\title{
Derivation of One-Sixth Hybrid Block Method for Solving General First Order Ordinary Differential Equations
}

\author{
Rufai M. A. ${ }^{1}$, Duromola M.K. ${ }^{2}$, and Ganiyu A.A. ${ }^{3}$ \\ ${ }^{I}$ Department of Mathematical Sciences, Federal University of Technology, Akure, Nigeria. \\ ${ }^{2}$ Department of Mathematical Sciences, Federal University of Technology, Akure, Nigeria. \\ ${ }^{3}$ Department of Mathematics, Adeyemi College of Education, Ondo, Ondo State, Nigeria.
}

\begin{abstract}
This paper focuses on the derivation of one-sixth hybrid block method for the general solution of first order initial values problems of ordinary differential equations. The new proposed method was derived by using the approach of collocation and interpolation of Chebyshev polynomials, approximate solution at some selected points to get a continuous linear multistep method, which was evaluated at some off-grid points to generate hybrid linear multistep methods. Basic properties of the proposed method wasexamined and the method found to be zero-stable, consistent and convergent. The efficiency of the method was tested on some numerical examples and in particular, on well-known SIR Model, Prothero-Robinson oscillatory problem and highly stiff oscillatory problem. On comparison, the new proposed method performed favourably when compare with the existing method proposed by other researchers in the area of Numerical Analysis.
\end{abstract}

Keywords: Hybrid block methods, first order initial values problems, collocation and interpolation, approximate solutions, consistent and zero stability.

\section{Introduction}

Ordinary differential equation can be found in almost all disciplines. For instance, mathematical models of electrical circuits, mechanical systems, chemical processes, biological processes to mention but a few. After the introduction of calculus, it was discovered that not all ordinary and partial differential equations can be solved analytically. In order to overcome this problem, numerical methods for solving ordinary differential equations were introduced with the aim of providing an approximate solution to the ordinary differential equations. Most famous ones are the Euler forward and Euler backward methods, Adams and Bash-forth, linear multistep methods, Runge-kutta, Heun methods and among other methods.

The numerical solution of general first order initial value problems of ordinary differential equation of the form:

$$
y^{\prime}=f(x, y), y\left(x_{0}\right)=y_{0}, y, x_{0}, f \varepsilon \Re,
$$

where $x_{0}$ represent initial point and $f$ is the continuous function and fulfills the Lipchitz's condition that verifies the uniqueness and existence theorem stated by Awoyemi (2011) is consider in this paper.

Many problems in form of equation (1) required a lot of attention from many researchers in the literature simply because of its applications in Chemistry, Engineering, Physics, Economics, Computer Sciences and Social Sciences. It has been verified that first order ordinary differential equations of the form equation (1) do not often have theoretical solution, hence seeking for numerical solution is very important. According to Dahlquist (1956) and Odekunle et al. (2012), equation (1) is used in simulating the growth of population, trajectory of a particle, simple harmonic motion, deflection of a beam and the like. It is also important to note that mixture models, SIR model, Prothero-Robinson oscillatory problem and highly stiff oscillatory problem and other similar problems can be written in the form of equation (1).

The desire to obtain more accurate approximate solutions to mathematical models arising from many disciplines, had led several scholars like; Lambert, (1991), Hairer, (1996), Badmus and Mishehia (2011), Adesanya et al. (2012), Fatokun et al. (2011), Zarina and Kharil (2005), Dahlquist (1963), Onumanyi et al. (1994) and among other scholars to propose different numerical methods. These authors proposed a multi-derivative method which is implemented in predictor-corrector mode. The major setback of this method is that the predictors are in decreasing order of accuracy; hence it affects the efficiency and accuracy of their methods.

Block methods for solving ordinary differential equations have been proposed and presented by some researchers such as: Areo et al. (2011), Ibijola (2011), Abbas (1997), Odekunle et al (2012), Yahaya (2007) and Mohammed and Yahaya (2010). Theseresearchers proposed block methods which has the characteristics of Runge-kutta method for being self-starting and does not require development of separate predictors or starting values.

The main goal of this paper is to derive a new continuous hybrid block method using Chebyshev polynomials of the first kind as basis function and the new proposed method will give solutions to initial value problems of linear, nonlinear and systems of first order ordinary differential equations. 


\section{Development of the One-Sixth Step Method}

In this section, the objective is to derive the hybrid block methods of the form:

$$
\begin{aligned}
& y_{n+\frac{1}{6}}-y_{n}=h\left[\sum_{j=0}^{\frac{1}{6}} \beta_{j}(x) f_{n+j}\right], \\
& y_{n+\frac{1}{9}}-y_{n}=h\left[\sum_{j=0}^{\frac{1}{6}} \beta_{j}(x) f_{n+j}\right], \\
& y_{n+\frac{1}{18}}-y_{n}=h\left[\sum_{j=0}^{\frac{1}{6}} \beta_{j}(x) f_{n+j}\right]
\end{aligned}
$$

where $j=0, \frac{1}{18}, \frac{1}{9}$, and $\frac{1}{6}, \beta_{j}$ represents continuous coefficients, $y_{n+j}=y\left(x_{n}+j h\right)$ is the numerical solution of the analytical solution at the point $x_{n+j}$ and $f_{n+j}=f \operatorname{left}\left(x_{n+j}, y_{n+j}\right.$.

In order to get equations(2), (3)and(4), we seek a Chebyshev polynomial of the first kind as a basis function to be of the form:

$$
Y(x)=\sum_{j=0}^{4} a_{j} T_{j}(x),
$$

where $a_{j}{ }^{\prime} s, j=0,1,2,3$ and 4 are unknown coefficients to be determined and $T_{j}(x)$ represent Chebyshev polynomial of the first kind of degree 4 .

The Chebyshev polynomial of the first kind is defined as follow:

$$
T_{j}(x)=\cos \left(\operatorname{jos}^{-1} x\right),-1 \leq x \leq 1
$$

Equation (6) satisfies the following triple recursion relation

The first derivative of the equation (5) is:

$$
T_{j}(x)=2 x T_{j}(x)-T_{j-1}(x), j \geq 1
$$

$$
Y^{\prime}(x)=\sum_{j=0}^{i+c-1} a_{j} T_{j}^{\prime}(x)
$$

In order to derive the schemes, equations (5) and (6) are interpolated and collocated at the points $x_{n+i}, i=0$ and $x_{n+c}, c=0, \frac{1}{18}, \frac{1}{9}$ and $\frac{1}{6}$ respectively to generate the following system of equations.

$$
\begin{gathered}
y_{n}=a_{0}-a_{2}+a_{4} \\
f_{n}=a_{1}-3 a_{3} \\
f_{n+\frac{1}{18}}=a_{1}+\frac{2}{9} a_{2}-\frac{80}{27} a_{3}-\frac{644}{729} a_{4} \\
f_{n+\frac{1}{9}}=a_{1}+\frac{4}{9} a_{2}-\frac{77}{27} a_{3}-\frac{1264}{729} a_{4} \\
f_{n+\frac{1}{6}}=a_{1}+\frac{2}{3} a_{2}-\frac{8}{3} a_{3}-\frac{68}{27} a_{4}
\end{gathered}
$$

The parameters $a_{0}, a_{1}, a_{2}, a_{3}, a_{4}$ are obtained by using Gaussian elimination method and presented as follow:

$$
\begin{gathered}
a_{0}=y_{n}+\frac{2295}{8} h f_{n+\frac{1}{18}}-\frac{2241}{8} h f_{n+\frac{1}{9}}+\frac{741}{8} h f_{n+\frac{1}{6}}-\frac{795}{8} h f_{n} \\
a_{1}=82 h f_{n}-\frac{81}{2} h f_{n+\frac{1}{6}}-\frac{405}{2} h f_{n+\frac{1}{18}}+162 h f_{n+\frac{1}{9}} \\
a_{2}=378 h f_{n+\frac{1}{18}}-\frac{1485}{4} h f_{n+\frac{1}{9}}+123 h f_{n+\frac{1}{6}}-\frac{519}{4} h f_{n} \\
a_{3}=-\frac{27}{2} h f_{n+\frac{1}{6}}+27 h f_{n}-\frac{135}{2} h f_{n+\frac{1}{18}}+54 h f_{n+\frac{1}{9}} \\
a_{4}=\frac{729}{8} h f_{n+\frac{1}{18}}-\frac{729}{8} h f_{n+\frac{1}{9}}+\frac{243}{8} h f_{n+\frac{1}{6}}-\frac{243}{8} h f_{n}
\end{gathered}
$$

On Substituting the value of $a_{0}, a_{1}, a_{2}, a_{3}, a_{4}$ into equation (5) gives the continuous scheme 


$$
\begin{aligned}
Y(x)= & 729 h x^{4} f_{n+\frac{1}{18}}+27 h x^{2} f_{n+\frac{1}{18}}-270 h x^{3} f_{n \frac{1}{18}}+y_{n}+108 h f_{n} x^{3} \\
+ & x h f_{n}-\frac{33}{2} h f_{n} x^{2}-243 h f_{n} x^{4}+216 h f_{n+\frac{1}{9}} x^{3}-\frac{27}{2} h f_{n+\frac{1}{9}} x^{2} \\
& -729 h f_{n+\frac{1}{9}} x^{4}+243 x^{4} h f_{n+\frac{1}{6}}-54 x^{3} h f_{n+\frac{1}{6}}+3 x^{2} h f_{n+\frac{1}{6}}
\end{aligned}
$$

Evaluating equation (19) at the non-interpolating points i.e $x=\frac{1}{18}, \frac{2}{18}, \frac{3}{18}$ give the following scheme:

$$
\begin{gathered}
y_{n+\frac{1}{18}}=\frac{19}{432} h f_{n+\frac{1}{18}}+y_{n}+\frac{1}{48} h f_{n}-\frac{5}{432} h f_{n+\frac{1}{9}}+\frac{1}{432} h f_{n+\frac{1}{6}} \\
y_{n+\frac{1}{9}}=\frac{2}{27} h f_{n+\frac{1}{18}}+y_{n}+\frac{1}{54} h f_{n}+\frac{1}{54} h f_{n+\frac{1}{9}} \\
y_{n+\frac{1}{6}}=\frac{1}{16} h f_{n+\frac{1}{18}}+y_{n}+\frac{1}{48} h f_{n}+\frac{1}{16} h f_{n+\frac{1}{9}}+\frac{1}{48} h f_{n+\frac{1}{6}}
\end{gathered}
$$

\subsection{Formulation of Block for the Proposed Method}

To get block for a new one-sixth step, we combine equations $(20-22)$ and we use their coefficients to form a block of the form:

$A^{0} Y_{m}=A^{1} y_{n}+h D f\left(y_{n}\right)+h E F\left(Y_{m}\right)$,
Where $\quad Y_{m}=\left[y_{n+\frac{1}{18}}, y_{n+\frac{1}{9}}, y_{n+\frac{1}{6}}\right]^{T}, \quad y_{n}=\left[y_{n-\frac{1}{6}}, \ldots, y_{n}\right]^{T}, \quad F\left(Y_{n}\right)=\left[f_{n+\frac{1}{18}}, \ldots, f_{n+\frac{1}{6}}\right]^{T}, \quad f\left(y_{n}\right)=$ $\left[f_{n-\frac{1}{6}}, \ldots, f_{n}\right]^{T}$

$$
\begin{aligned}
A^{0} & =\left[\begin{array}{lll}
1 & 0 & 0 \\
0 & 1 & 0 \\
0 & 0 & 1
\end{array}\right], \\
A^{1} & =\left[\begin{array}{lll}
0 & 0 & 1 \\
0 & 0 & 1 \\
0 & 0 & 1
\end{array}\right], \\
D & =\left[\begin{array}{lll}
0 & 0 & \frac{1}{48} \\
0 & 0 & \frac{1}{54} \\
0 & 0 & \frac{1}{48}
\end{array}\right], \\
E & =\left[\begin{array}{ccc}
\frac{19}{432} & -\frac{5}{432} & \frac{1}{432} \\
\frac{2}{27} & \frac{1}{54} & 0 \\
\frac{1}{16} & \frac{1}{16} & \frac{1}{48}
\end{array}\right] .
\end{aligned}
$$

\section{Analysis of the Basic Properties of the Proposed Methods}

Here, we consider the analysis of the basic properties of the proposed method which includes: zero stability, order, error constants, consistency and convergence of the methods.

\subsection{Order and Error Constant of the Block Methods}

Following the method proposed by Areo and Rufai (2016) and Areo and Omojola (2015), we represented the linear difference operator as follow:

$$
L[y(x) ; h]=\sum_{j=0}^{k}\left[\alpha_{j} y(x+j h)-h \beta_{j} y^{\prime}(x+j h)\right] .
$$

On expanding equation (24) using Taylor series and comparing their coefficients, we get the following equation. where

$$
L[y(x) ; h]=C_{0} y(x)+C_{1} h y^{\prime}(x)+C_{1} h^{2} y^{\prime \prime}(x) \ldots+C_{q} h^{q} y^{q}(x),
$$

$$
C_{q}=\frac{1}{q !}\left[\sum_{j=1}^{k} j^{q} \alpha_{j}-q \sum_{j=1}^{k} j^{q-1} \beta_{j}\right], \text { where } q=0,1,2, \ldots n .
$$

\section{Definition:}

The linear difference operator and the associated block method are said to be of order $p$, if $C_{0}=C_{1}=\ldots=C_{p}=$ 
$0, C_{p+1} \neq 0$, where $C_{p+1}$ denoted the error constant.

By carrying out Taylor series expansion on equations $(16-18)$ we get:

When $n=0$, we get:

$$
C_{n}=\left[\begin{array}{c}
\frac{\left(\frac{1}{18}\right)^{n}}{n !}-\frac{\left(\frac{19}{432}\left(\frac{1}{18}\right)^{n-1}-\frac{5}{432}\left(\frac{1}{9}\right)^{n-1}+\frac{1}{432}\left(\frac{1}{6}\right)^{n-1}\right)}{(n-1) !} \\
\frac{\left(\frac{1}{9}\right)^{n}}{n !}-\frac{\left(\frac{2}{27}\left(\frac{1}{18}\right)^{n-1}+\frac{1}{54}\left(\frac{1}{9}\right)^{n-1}\right)}{(n-1) !} \\
\frac{\left(\frac{1}{6}\right)^{n}}{n !}-\frac{\frac{1}{16}\left(\frac{1}{18}\right)^{n-1}+\frac{1}{16}\left(\frac{1}{9}\right)^{n-1}+\frac{1}{48}\left(\frac{1}{6}\right)^{n-1}}{(n-1) !}
\end{array}\right]
$$

$$
C_{0}=\left[\begin{array}{l}
0 \\
0 \\
0
\end{array}\right]
$$

When $n=1$, we get:

$$
C_{1}=\left[\begin{array}{l}
0 \\
0 \\
0
\end{array}\right]
$$

When $n=2$, we get:

$$
C_{2}=\left[\begin{array}{l}
0 \\
0 \\
0
\end{array}\right]
$$

When $n=3$, we get:

$$
C_{3}=\left[\begin{array}{l}
0 \\
0 \\
0
\end{array}\right]
$$

When $n=4$, we get:

$$
C_{4}=\left[\begin{array}{l}
0 \\
0 \\
0
\end{array}\right]
$$

When $n=5$, we get:

$$
C_{5}=\left[\begin{array}{c}
-\frac{19}{1360488960} \\
-\frac{1}{170061120} \\
-\frac{1}{50388480}
\end{array}\right]
$$

Hence, the proposed block methods are of order $p=(4,4,4)$ with error constants $\left(-\frac{19}{1360488960},-\frac{1}{170061120},-\frac{1}{50388480}\right)$.

\subsection{Zero Stability of the Block Method}

A block method (23) is said to be zero stable if the roots $\left|\left[\lambda A^{(0)}-A^{(1)}\right]\right|=0$

To show that $\left|\left[\lambda A^{(0)}-A^{(1)}\right]\right|=0$, we have:

This implies that $\lambda^{3}-\lambda^{2}=0, \lambda=0,0,1$

$$
\left|\left[\lambda A^{(0)}-A^{(1)}\right]\right|=\left|\left[\lambda\left(\begin{array}{lll}
1 & 0 & 0 \\
0 & 1 & 0 \\
0 & 0 & 1
\end{array}\right)-\left(\begin{array}{lll}
0 & 0 & 1 \\
0 & 0 & 1 \\
0 & 0 & 1
\end{array}\right)\right]\right|=0
$$

Since all roots $(\lambda)$ have modulus less than or equal to one and $|\lambda|=1$ is simple. Hence the proposed method is zero-stable.

\subsection{Consistency and Convergence of the Proposed Method}

According to Areo and Rufai (2016), if the order of a linear hybrid multistep method is greater than or equal to one i.e. $(p \geq 1)$, the method is consistency, since our new proposed methods are of constant order $p=4$. Therefore, our new proposed method is consistent. According to Jator (2007), the two sufficient conditions for a linear hybrid multistep method to be convergent are to be zero-stable and consistent. Since the two conditions are satisfied. Hence the new derived method converges. 


\section{Numerical Experiments}

In this section, we deals with the implementation of the derived methods in solving initial value problems of first order ordinary differential equations. The methods were coded using Maple 17 environment.

\subsection{Problem 1: SIR Model}

The SIR model is an epidemiological model that computes the theoretical number of people infected with a contagious illness in a closed population over time. The name of this class of models derives from the fact that they involve coupled equations relating the number of susceptible people $S(t)$, number of people infected $I(t)$ and the number of people who have recovered $R(t)$. This is a good and simple model for many infectious diseases including measles, mumps and rubella see [Gragg and Stetter (1964), Rosser (1967) and Mickens (1994)]. It is given by the following three coupled equations,

$$
\begin{gathered}
\frac{d S}{d t}=\mu(1-S)-\beta I S, \\
\frac{d I}{d t}=\mu I-\gamma I+\beta I S, \\
\frac{d R}{d t}=-\mu R+\gamma I,
\end{gathered}
$$

where $\mu, \gamma$ and $\beta$ are positive parameters. Define $y$ to be

$$
y=S+I+R
$$

By adding equations(23), (24)and(25), we obtain the following evolution equation for $y$ as follow;

$$
y^{\prime}=\mu(1-y)
$$

Taking $\mu=0.5$ and attaching an initial condition $y(0)=0.5$ (for a particular closed population), we obtain

Whose exact solution is $1-0.5 e^{0.5 t}$.

$$
y^{\prime}(t)=0.5(1-y), y(0)=0.5
$$

Source:[Sunday et. al. 2013].

By applying the hybrid block method (23) on problem (1), we obtain the results in table (1) at different values of time (t).

Table 1. Performance of the block method (23) on problem 1

\begin{tabular}{ccccl}
\hline \hline t-value & Numerical Results & Exact Results & Error in (23) & Error in Sunday et al. \\
\hline 0.1000 & 0.524385287749660 & 0.524385287749643 & $1.714000 \mathrm{E}-14$ & $5.574430 \mathrm{E}-012$ \\
0.2000 & 0.547581290982053 & 0.547581290982020 & $3.260000 \mathrm{E}-14$ & $3.946177 \mathrm{E}-012$ \\
0.3000 & 0.569646011787518 & 0.569646011787471 & $4.653000 \mathrm{E}-14$ & $8.183232 \mathrm{E}-012$ \\
0.4000 & 0.590634623461068 & 0.590634623461009 & $5.902000 \mathrm{E}-14$ & $3.436118 \mathrm{E}-011$ \\
0.5000 & 0.610599608464368 & 0.610599608464298 & $7.018000 \mathrm{E}-14$ & $1.929743 \mathrm{E}-010$ \\
0.6000 & 0.629590889659221 & 0.629590889659141 & $8.011000 \mathrm{E}-14$ & $1.879040 \mathrm{E}-010$ \\
0.7000 & 0.647655955140732 & 0.647655955140643 & $8.891000 \mathrm{E}-14$ & $1.776835 \mathrm{E}-010$ \\
0.8000 & 0.664839976982277 & 0.664839976982180 & $9.665000 \mathrm{E}-14$ & $1.724676 \mathrm{E}-010$ \\
0.9000 & 0.681185924189217 & 0.681185924189113 & $1.034200 \mathrm{E}-13$ & $1.847545 \mathrm{E}-010$ \\
1.0000 & 0.696734670143793 & 0.696734670143683 & $1.093100 \mathrm{E}-13$ & $3.005770 \mathrm{E}-010$ \\
\hline
\end{tabular}

\subsection{Problem 2:}

We consider a mildly stiff system problem which was also solved by Yakubu and Markus (2016).

$$
\left[\begin{array}{l}
y_{1}^{\prime}(x) \\
y_{2}^{\prime}(x)
\end{array}\right]=\left[\begin{array}{cc}
998 & 1998 \\
-999 & -1999
\end{array}\right]\left[\begin{array}{l}
y_{1}(x) \\
y_{2}(x)
\end{array}\right] \text {, with initial condition, }\left[\begin{array}{l}
y_{1}(x) \\
y_{2}(x)
\end{array}\right]=\left[\begin{array}{l}
1 \\
1
\end{array}\right] h=0.1
$$

Exact solution:

$$
y_{1}(x)=4 e^{-x}-3 e^{-1000 x}, y_{2}(x)=-2 e^{-x}+3 e^{-1000 x}
$$

Table 2: Performance of the block method (23) on problem 2 
Derivation of One-Sixth Hybrid Block Method for Solving General First Order Ordinary Differential...

\begin{tabular}{ccll}
\hline \hline$x$ & $y_{i}$ & Error in $(23)$ & Error in Yakubu and Markus \\
\hline 5 & $y_{1}$ & $1.392000000000000 \times 10^{-11}$ & $1.58384223934188 \times 10^{-2}$ \\
& $y_{2}$ & $6.970000000000000 \times 10^{-12}$ & $7.91952513657462 \times 10^{-3}$ \\
40 & $y_{1}$ & $3.362800000000000 \times 10^{-12}$ & $3.81292881577727 \times 10^{-7}$ \\
& $y_{2}$ & $1.681800000000000 \times 10^{-12}$ & $1.90646440788863 \times 10^{-7}$ \\
70 & $y_{1}$ & $2.93250000000000 \times 10^{-13}$ & $8.90990527186305 \times 10^{-12}$ \\
& $y_{2}$ & $1.466400000000000 \times 10^{-13}$ & $4.45495263593152 \times 10^{-12}$ \\
\hline
\end{tabular}

\subsection{Problem 3:}

We consider the highly stiff of ordinary differential equation which was also solved by Skwaneet. al. (2013).

$y^{\prime}=-\alpha\left(y_{0}-F(x)\right)+F^{\prime}(x)$, with initial condition $y\left(x_{0}\right)=y_{0}, x \in[0,0.1], h=0.01$,

Exact solution:

$$
y(x)=y_{0}+\exp (-\alpha x)-1, \quad \text { where } \alpha=10, F(x)=0, x_{0}=0 \text { and } y_{0}=1 .
$$

Table 3:Performance of the block method (23) on problem 3

\begin{tabular}{ccccl}
\hline \hline x-value & Numerical Results & Exact Results & Error in (23) & Error in Skwane et al. \\
\hline 0.0100 & 0.904837418034880 & 0.904837418035960 & $1.079154 \mathrm{E}-12$ & $2.323184 \mathrm{E}-07$ \\
0.0200 & 0.818730753076029 & 0.818730753077982 & $1.952918 \mathrm{E}-12$ & $1.006706 \mathrm{E}-07$ \\
0.0300 & 0.740818220679067 & 0.740818220681718 & $2.650610 \mathrm{E}-12$ & $3.250567 \mathrm{E}-07$ \\
0.0400 & 0.670320046032441 & 0.670320046035639 & $3.197828 \mathrm{E}-12$ & $4.662291 \mathrm{E}-07$ \\
0.0500 & 0.606530659709017 & 0.606530659712633 & $3.616893 \mathrm{E}-12$ & $3.407125 \mathrm{E}-07$ \\
0.0600 & 0.548811636090099 & 0.548811636094026 & $3.927240 \mathrm{E}-12$ & $4.816158 \mathrm{E}-07$ \\
0.0700 & 0.496585303787264 & 0.496585303791410 & $4.145766 \mathrm{E}-12$ & $5.632829 \mathrm{E}-07$ \\
0.0800 & 0.449328964112934 & 0.449328964117222 & $4.287136 \mathrm{E}-12$ & $4.495628 \mathrm{E}-07$ \\
0.0900 & 0.406569659736235 & 0.406569659740599 & $4.364056 \mathrm{E}-12$ & $5.351845 \mathrm{E}-07$ \\
0.1000 & 0.367879441167055 & 0.367879441171442 & $4.387513 \mathrm{E}-12$ & $5.787084 \mathrm{E}-07$ \\
\hline
\end{tabular}

\subsection{Problem 4:}

We consider the Prothero-Robinson Oscillatory ordinary differential equation which was also solved by Sunday et al. (2014).

$y^{\prime}=-\alpha\left(y_{0}-F(x)\right)+F^{\prime}(x)$, with initial condition $y\left(x_{0}\right)=y_{0}, x \in[0,0.1], h=0.01$,

Exact solution:

$$
y(x)=y_{0}+\exp (-\alpha x)-1, \quad \text { where } \alpha=10, F(x)=0, x_{0}=0 \text { and } y_{0}=1 .
$$

Table 4: Performance of the block method (23) on problem 4

\begin{tabular}{ccccl}
\hline \hline x-value & Numerical Results & Exact Results & Error in (23) & Error in Sunday et al. \\
\hline 0.1000 & 0.099833416647961 & 0.099833416646828 & $1.133052 \mathrm{E}-12$ & $2.000000 \mathrm{E}-11$ \\
0.2000 & 0.198669330797208 & 0.198669330795061 & $2.146863 \mathrm{E}-12$ & $3.000000 \mathrm{E}-11$ \\
0.3000 & 0.295520206664381 & 0.295520206661340 & $3.041572 \mathrm{E}-12$ & $1.000000 \mathrm{E}-10$ \\
0.4000 & 0.389418342312468 & 0.389418342308650 & $3.817533 \mathrm{E}-12$ & $2.000000 \mathrm{E}-10$ \\
0.5000 & 0.479425538608678 & 0.479425538604203 & $4.475401 \mathrm{E}-12$ & $1.000000 \mathrm{E}-10$ \\
0.6000 & 0.564642473400052 & 0.564642473395035 & $5.016211 \mathrm{E}-12$ & $2.000000 \mathrm{E}-10$ \\
0.7000 & 0.644217687243132 & 0.644217687237691 & $5.441444 \mathrm{E}-12$ & $1.000000 \mathrm{E}-10$ \\
0.8000 & 0.717356090905276 & 0.717356090899523 & $5.753080 \mathrm{E}-12$ & $2.000000 \mathrm{E}-10$ \\
0.9000 & 0.783326909633437 & 0.783326909627483 & $5.953640 \mathrm{E}-12$ & $3.000000 \mathrm{E}-10$ \\
1.0000 & 0.841470984813943 & 0.841470984807897 & $6.046223 \mathrm{E}-12$ & $3.000000 \mathrm{E}-10$ \\
\hline
\end{tabular}

\subsection{Problem 5:}

We consider a nonlinear first order ordinary differential problem which was also solved by Okunuga et al. (2013).

$$
y^{\prime}=-10(y-1)^{2}, \text { with initial condition } y(0)=2, x \in[0,0.1], h=0.01
$$


Exact solution:

$$
y(x)=1+\frac{1}{1+10 x} .
$$

Table 5: Performance of the block method (23) on problem 5

\begin{tabular}{cccll}
\hline \hline x-value & Computed Result & Exact Result & Error in Method (19) & Error in Okunuga et al. (2013) \\
0.0100 & 1.9090909090909092 & 1.9090924673408327 & $1.558250 \mathrm{E}-06$ & $1.070000 \mathrm{E}-03$ \\
0.0200 & 1.8333333333333335 & 1.8333357330822146 & $2.399749 \mathrm{E}-06$ & $2.380000 \mathrm{E}-03$ \\
0.0300 & 1.7692307692307692 & 1.7692335996830317 & $2.830452 \mathrm{E}-06$ & $2.210000 \mathrm{E}-03$ \\
0.0400 & 1.7142857142857144 & 1.7142887352245348 & $3.020939 \mathrm{E}-06$ & $5.360000 \mathrm{E}-03$ \\
0.0500 & 1.6666666666666672 & 1.6666697362310798 & $3.069564 \mathrm{E}-06$ & $7.530000 \mathrm{E}-03$ \\
0.0600 & 1.6250000000000007 & 1.6250030345684419 & $3.034568 \mathrm{E}-06$ & $9.000000 \mathrm{E}-03$ \\
0.0700 & 1.5882352941176476 & 1.5882382452642667 & $2.951147 \mathrm{E}-06$ & $9.980000 \mathrm{E}-03$ \\
0.0800 & 1.5555555555555558 & 1.5555583964373967 & $2.840882 \mathrm{E}-06$ & $1.060000 \mathrm{E}-02$ \\
0.0900 & 1.5263157894736841 & 1.5263185065995473 & $2.717126 \mathrm{E}-06$ & $1.100000 \mathrm{E}-02$ \\
0.1000 & 1.4999999999999998 & 1.5000025881572263 & $2.588157 \mathrm{E}-06$ & $1.120000 \mathrm{E}-03$ \\
\hline
\end{tabular}

\section{Discussion of the Results}

In this manuscript, we have applied the procedure of collocation and interpolation to develop one-sixth hybrid block method for solving general first order ordinary differential equations. In the table 1, table $\mathbf{2}$ and table 3, it is shown that our new method is more accurate than the methods proposed by Sunday et al. (2013), Yakubu and Markus (2016) and Skwane et al. (2013). It has been seen from table $\mathbf{4}$ and table 5 that our new method yield better results than the results presented by Sunday etal. (2014) and Okunuga et al. (2013), despite the high order of their methods, our new hybrid block method of order four are more efficient and accurate than their methods of order seven.

\section{Conclusion}

We have proposed a new hybrid block method that gives solutions to first order initial value problems of first order ordinary differential equations. We used our new derived schemes to solve some numerical problems and the results obtained were significantly better when compared with those in the tables $\mathbf{1}, \mathbf{2}, \mathbf{3}, \mathbf{4}$, and $\mathbf{5}$. We conclude that the new method gives better approximate solutions than some existing methods.

\section{References}

[1] Abbas S., Derivations of New Block Method for the Numerical Solution of First Order IVPs, International Journal of Computer Mathematics, Vol. 64, (1997), pp. 11-25.

[2] Adesanya A. O., Odekunle M. R. and James A. A., Starting Hybrid Stomer-Cowell More Accurately by Hybrid Adams Method for the Solution of First Order Ordinary Differential Equation, European Journal of Scientific Research, Vol. 77 No. 4, (2012), pp. 580-588.

[3] Areo E. A., Ademiluyi R. A. and Babatola P. O. , Three-Step Hybrid Linear Multistep Method for the Solution of First Order Initial Value Problems in Ordinary Differential Equations, J.N.A.M.P, Vol. 19, (2011), pp. 261-266.

[4] Areo, E.A. and Omojola, M. O., A New One-Twelfth Step Continuous Block Method for the Solution of Modeled Problems of Ordinary Differential Equation, American Journal of Computational Mathematics, Volume 5 (2015), pp. $447-457$.

[5] Areo, E.A. and Adeniyi R. B. (2013), "A Self-Starting Linear Multistep Method for Direct Solution of Second Order Differential Equations", International Journal of Pure and Applied Mathematics, Bulgaria, Vol. 82, No. 3 pp. 345-364.

[6] Areo, E.A. and Rufai M.A.(2016), A New Uniform Fourth Order One-Third Step Continuous Block Method for Direct Solutions of $y^{\prime \prime}=f(x, y, y)$. British Journal of Mathematics and Computer Science, Science Domain International, vol. 15(4), page1 -12.

[7] Awoyemi D.O, Adebile E.A, Adesanya A.O, Anake T.A., Modified block method for the direct solution of second order ordinary differential equations. Intern. J. Applied Mathematics and Computation 3, (2011), pp. 181-188.

[8] Badmus A. M. and Mishehia D. W., Some Uniform Order Block Methods for the Solution of First Ordinary Differential Equation, J. N.A.M. P, Vol. 19, (2011), pp 149- 154.

[9] Dahlquist, G. G. Numerical integration of ordinary differential equations, Math. Scand., 4 (1956), pp. 33-50.

[10] Dahlquist G., A Special Stability Problem for Linear Multistep Methods, BIT, Vol. 3, (1963), pp. $27-43$.

[11] Fatokun J., Onumanyi P. and Sirisena U. W., Solution of First Order System of Ordering Differential Equation by Finite Difference Methods with Arbitrary, J.N.A.M.P, (2011), pp. 30-40.

[12] Fatunla S. O., Block methods for second order IVPs, Int.J. Comput.Maths., 41, (1991) 55-63.

[13] Gragg W. B. and Stetter H. J. Generalized Multistep Predictor-Corrector Methods, Journal of Association of Computing Machines, Vol. 11, No. 2, (1964), pp. 188-209.

[14] Hairer E., Wanner G., Solving Ordinary Differential Equations II, Stiff and Differential-Algebraic Problems. Second Revised Edition, Springer-Series in Computational Mathematics, 14,(1996), pp. 75-77.

[15] Ibijola E. A., Skwame Y. and Kumleng G. , Formation of Hybrid of Higher Step-Size, through the Continuous Multistep Collocation, American Journal of Scientific and Industrial Research, Vol. 2, (2011), pp. 161-173.

[16] Jator, S. N., A sixth order linear multistep method for the direct solution of $y^{\prime \prime}=f\left(x, y, y^{\prime}\right)$. International journal of pure and applied Mathematics, 40(1), (2007), pp. 457-472.

[17] Mohammed U. and Yahaya Y. A. , Fully implicit four point block backward difference formula for solving first-order initial value problems, Leonardo Journal of Sciences, 16(2010), pp. 21-30.

[18] Mickens R. E. Nonstandard Finite Difference Models of Differential Equations, World Scientific, Singapore, (1994).

[19] Okunuga S. A., Sofoluwe A. B. and Ehigie J. O., Some block numerical schemes for solving initial value problems in ODEs. Journal of Mathematical Sciences. (2013), 2(1), 387-402.

[20] Onumanyi P. Awoyemi D.O. Jator S.N., Sirisena U.W., New linear multistep methods with continuous coefficients for first order initial value problems. J Niger Math Society (1994) vol 13, pp. 37-51.

[21] Rosser J. B., A Runge-Kutta Method for All Seasons, SIAM Review, Vol. 9, No. 3, (1967), pp. 417-452.

[22] Skwane Y., Sunday J. and Odekunle M. R., A Continues Block Method Integrator for the Solution of Stiff and Oscillatory Differential 
Equations, IOSR Journal of Mathematics, 8(3), (2013) pp. 75-80.

[23] Sunday J. ,Odekunle M. R. and Adeyanya A. O., Order Six Block Integrator for the Solution of First-Order Ordinary Differential Equations, IJMS, Vol. 3, No. 1 (2013), pp. 87-96.

[24] Sunday J, Adesanya A.O., Odekunle M.R. A self- starting four- step fifth- order block integrator for stiff and oscillatory differential equations. Journal of Mathematical and Computational Sciences. (2014), 4(1), pp. 73-84.

[25] Yahaya Y. A. and Kimleng G. M., Continuous of Two-Step Method with Large Region of Absolute Stability, J.N.A.M.P,(2007), pp. 261-268.

[26] Yakubu D. G. and Markus S., Second derivative of high-order accuracy methods for the numerical integration of stiff initial value problems, Afr. Mat. Springer Journal, (2016).

[27] Zarina B. I. and Kharil S. I., Block Method for Generalized Multistep Method Adams and Backward Differential Formulae in Solving First Order ODEs, MATHEMATIKA, (2005), pp. 25-33. 